\title{
Constraints on the box-shaped cosmic ray electron feature from dark matter annihilation with the AMS-02 and DAMPE data
}

\author{
Lei Zu, ${ }^{1,2}$ Cun Zhang, ${ }^{1,3}$ Lei Feng, ${ }^{1, *}$ Qiang Yuan, ${ }^{1,2, \dagger}$ and Yi-Zhong Fan ${ }^{1,2, \dagger}$ \\ ${ }^{1}$ Key Laboratory of Dark Matter and Space Astronomy, Purple Mountain Observatory, \\ Chinese Academy of Sciences, Nanjing 210008, China \\ ${ }^{2}$ School of Astronomy and Space Science, University of Science and Technology of China, \\ Hefei 230026, Anhui, China \\ ${ }^{3}$ School of Physics, Nanjing University, Nanjing 210092, China
}

(Received 6 December 2017; revised manuscript received 10 August 2018; published 19 September 2018)

\begin{abstract}
Precise measurements of spectra of cosmic ray electrons and positrons can effectively probe the nature of dark matter (DM) particles. In a class of models where DM particles initially annihilate into a pair of intermediate particles which then decay into standard model particles, box-shaped spectra can be generated. Such spectra are distinct from astrophysical backgrounds and can probably be regarded as characteristic features of the DM annihilation. In this work, we search for such a feature in the total electron plus positron spectrum measured by AMS-02 and DAMPE. No significant evidence for such a DM annihilation component has been found. The $95 \%$ confidence level upper limits of the velocity-weighted annihilation cross section are derived, which range from $\sim 10^{-26} \mathrm{~cm}^{3} \mathrm{~s}^{-1}$ for DM mass of $50 \mathrm{GeV}$ to $\sim 10^{-23} \mathrm{~cm}^{3} \mathrm{~s}^{-1}$ for DM mass of $10 \mathrm{TeV}$.
\end{abstract}

DOI: $10.1103 /$ PhysRevD.98.063010

\section{INTRODUCTION}

Dark matter (DM) is one of the very important unresolved mysteries in modern physics and astrophysics. The leading candidate of DM particle is the so-called weakly interacting massive particle (WIMP). Experiments with collider detection $[1,2]$, direct detection $[3,4]$ and indirect detection $[5,6]$ have been carried out to search for WIMPs. The indirect detection method aims to identify the relics in cosmic rays and/or gamma-rays from the annihilation or decay of DM [7-10]. Recent experiments such as PAMELA [11] and AMS-02 [12,13] discovered significant excess of cosmic ray positrons on top of the conventional background model prediction, which stimulated extensive discussion on the possible DM origin. At almost the same time, excesses in the total electron plus positron spectrum were also reported by several experiments [14-16], which may have a common origin with the positron anomaly.

\footnotetext{
* Corresponding author. fenglei@pmo.ac.cn

Corresponding author. yuanq@pmo.ac.cn

Corresponding author. yzfan@pmo.ac.cn

Published by the American Physical Society under the terms of the Creative Commons Attribution 4.0 International license. Further distribution of this work must maintain attribution to the author(s) and the published article's title, journal citation, and DOI. Funded by SCOAP ${ }^{3}$.
}

The observations of the antiproton fluxes are, however, largely consistent with the background model prediction [17-19] (see, however, [20,21]).

The DM annihilation/decay models [22-30] or new astrophysical sources [31-40] have been proposed to interpret the electron and positron excesses. For DM annihilation models, the annihilation cross section needs to be about $10^{-23} \mathrm{~cm}^{3} \mathrm{~s}^{-1}$, which is too large to account for the observed relic density unless a large boost factor $\left(\sim 10^{3}\right)$ is introduced [24,41]. For decaying DM models, the lifetime of DM particles is about $10^{26} \mathrm{~s}$. However, both scenarios have already been stringently constrained by the current multiwavelength observationals [42-48].

With proper assumptions of the background contribution, the electron and positron data can instead be used to constrain model parameters of DM, in particular, for those models with very distinct spectral features which are obviously missing in the data [49]. In this work, we focus on the specific scenario that DM particles annihilate into intermediate particle pairs which then decay into standard model particles [50,51]. Such a scenario is well motivated and can provide a large boost factor [24,41]. It can also produce a box-shaped spectrum to get distinguished from other astrophysical process effectively. This model has been adopted to interpret the possible excess of the AMS-02 antiproton data at $\sim 400 \mathrm{GeV}$ [52]. The box-shaped $\gamma$-ray feature was also investigated with the Fermi-LAT data [53,54].

Here we search for such spectral feature in the cosmic ray electron (and/or positron) spectrum. In particular, we will 
employ the most recent precise measurement of the total electron (i.e., electron plus positron) spectrum by the Dark Matter Particle Explorer (DAMPE; $[55,56])$. DAMPE is a high-energy cosmic ray particle detector with unprecedentedly high energy resolution and an excellent hadron rejection capability $[55,56]$. It can, therefore, measure the total electron spectrum with high precision, low background, and small systematic uncertainty. Recently, the DAMPE Collaboration published the first result about the measurement of the electron plus positron spectrum from $25 \mathrm{GeV}$ to $4.6 \mathrm{TeV}$ [57]. The wide energy coverage and high precision of the data can significantly improve the constraints on the properties of DM particles [58].

This work is organized as follows: In Sec. II, we present the spectrum of electrons generated by the two-step cascade annihilation of DM. In Sec. III, we briefly describe the propagation and background of electrons and positrons. In Sec. IVA, we assume the annihilation takes place in the whole Milky Way halo and perform the search for such signal in the AMS-02 and DAMPE data. In Sec. IV B we examine the scenario of the presence of a local DM subhalo. We summarize our results with some discussion in Sec. IV.

\section{BOX-SHAPED SPECTRA}

We focus on the two-step annihilation model, in which DM particles first annihilate into a pair of scalar particles $\phi$ which then decay into electrons and positrons. In the rest frame of $\phi$, the energy of the final electrons and positrons is $E_{e^{ \pm}}=m_{\phi} / 2$. Since we focus on high-energy cosmic rays in this work, it is reasonable to assume that $m_{e} \ll m_{\phi}<m_{\mathrm{DM}}$, where $m_{\mathrm{DM}}$ is the mass of DM particles. If the mass of $\phi$ is comparable with $m_{\mathrm{DM}}$ or $2 m_{e}$, a narrow peak spectrum of final state electrons and positrons would be produced [52]. In the lab frame, DM particles are nonrelativistic and $E_{\phi} \approx m_{\mathrm{DM}}$; hence, the energy of electrons or positrons is

$$
E=\frac{m_{\phi}^{2}+\beta \cos \theta \sqrt{4 m_{\mathrm{DM}}^{2} m_{e}^{2}\left(\beta^{2} \cos ^{2} \theta-1\right)+m_{\phi}^{4}}}{2 m_{\mathrm{DM}}\left(1-\beta^{2} \cos ^{2} \theta\right)},
$$

where $\beta=\sqrt{1-m_{\phi}^{2} / m_{\mathrm{DM}}^{2}}, \theta$ is the angle between the outgoing electron/positron and the parent scalar in the lab frame. For scalar intermediator $\phi$, the distribution of outgoing angles of electrons/positrons is isotropic in the rest frame of $\phi$. Therefore the electrons/positrons would have a box-shaped spectrum in the lab frame as

$$
\frac{d N}{d E}=\frac{2}{\sqrt{\left(m_{\mathrm{DM}}^{2}-m_{\phi}^{2}\right)\left(1-4 m_{e}^{2} / m_{\phi}^{2}\right)}} \Theta\left(E-E_{-}\right) \Theta\left(E_{+}-E\right),
$$

where $\Theta$ is Heaviside step function and $E_{ \pm}=m_{\mathrm{DM}} / 2 \pm$ $\sqrt{\left(m_{\mathrm{DM}}^{2}-m_{\phi}^{2}\right)\left(m_{\phi}^{2}-4 m_{e}^{2}\right)} / 2 m_{\phi}$. Our results reduce to that of Refs. [50,51] if we set $d N / d E_{\text {rest }}=\delta\left(E_{\text {rest }}-m_{\phi} / 2\right)$ in the rest frame of the intermediator. If we set $m_{e}=0$, Eq. (2) gives the photon spectrum due to the two-step annihilation process [53]. Note that we have neglected the electroweak corrections on the electron spectrum because its effect is tiny. In Ref. [59], the authors calculated the full spectrum with the electroweak corrections using the PYTHIA package [60], assuming a light $\mathrm{MeV}$-scale intermediate particle. We will compare our results with theirs (labeled as "PPPC4") in the following.

There are two free parameters $\left(m_{\mathrm{DM}}\right.$ and $\left.m_{\phi}\right)$ determining the spectrum. In the following we adopt $m_{\mathrm{DM}}$ and the mass ratio $m_{\phi} / m_{\mathrm{DM}}$ to present the results. If $m_{\phi} / m_{\mathrm{DM}} \sim 1$, the result reduces to a line spectrum. On the other hand, if $m_{\phi} / m_{\mathrm{DM}} \ll 1$ (but still have $m_{\phi} \gg m_{e}$ ), the produced boxshaped spectrum will be quite broad. For illustration we show in Fig. 1 the spectra of electrons for $m_{\mathrm{DM}}=1 \mathrm{TeV}$ and mass ratios of $(0.1,0.5,0.9)$, respectively. It is clear to show that the spectrum is broader for smaller mass ratio. The result from PPPC4 (with $m_{\mathrm{DM}}=1 \mathrm{TeV}$ ) is also presented in Fig. 1. The PPPC4 spectrum [59] is a numerical result with a light mediator including the soft photon radiation process and the high order corrections. Its general shape is indeed boxlike though it has wiggles.

\section{PROPAGATION OF ELECTRONS AND THE BACKGROUNDS}

\section{A. Propagation of cosmic ray electrons in the Galaxy}

Cosmic ray electrons and positrons propagate diffusively in the Galaxy, with significant cooling due to synchrotron and inverse Compton radiation processes. There are some

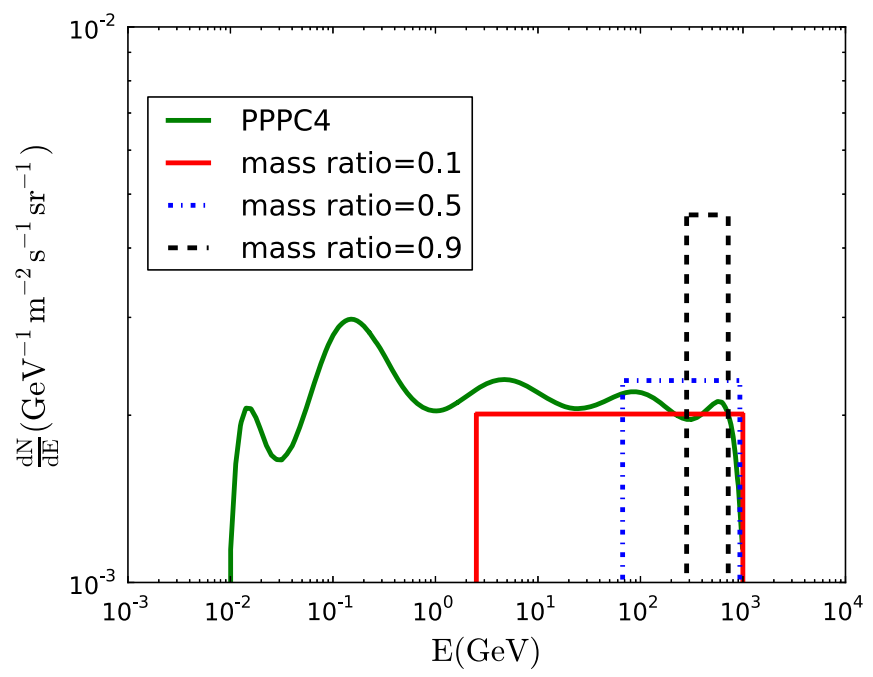

FIG. 1. Energy spectra of $e^{+} e^{-}$at production for different mass ratios between $m_{\phi}$ and $m_{\mathrm{DM}}$. Also shown is that from PPPC4 (green line). 
numerical tools to compute the propagation of cosmic rays, such as GALPROP [61] and DRAGON [62]. Here we adopt the LIKEDM package [63], which is essentially equivalent to GALPROP but employs a Green's function method (i.e., with quasimonochromatic injection spectrum at a series of energy grids) based on numerical tables obtained with GALPROP for given spatial distribution of the sources, to deal with the propagation of cosmic rays. This method has been verified to give good approximation to the GALPROP output, but is much more efficient [63].

The propagation is assumed to work in a diffusion reacceleration framework, with propagation parameters being determined by the boron-to-carbon ratio data and the Fermi diffuse $\gamma$-ray emission [64]. The main propagation parameters are: the diffusion coefficient $D(E)=$ $\beta D_{0}(E / 4 \mathrm{GeV})^{\delta}$ with $D_{0}=5.3 \times 10^{28} \mathrm{~cm}^{2} \mathrm{~s}^{-1}$ and $\delta=0.33$, the half-height of the propagation cynlinder $z_{h}=4 \mathrm{kpc}$, the Alfvenic speed which characterizes the reacceleration effect $v_{A}=33.5 \mathrm{~km} \mathrm{~s}^{-1}$. This set of propagation parameters is widely adopted in literature as the canonical "medium" one. Since high energy electrons and positrons can only travel a limited distance before cooled down, we expect that choosing other propagation parameters with different $z_{h}$ values would not affect the results significantly.

\section{B. Backgrounds}

The astrophysical background includes the conventional primary electrons from e.g., supernova remnants, and secondary electrons and positrons from the inelastic collisions between cosmic ray nuclei and the interstellar medium. Additional astrophysical sources, in particular pulsars, are also expected to be high-energy electron/ positron sources [65], and may be responsible for the positron anomaly [31-33]. In this work we discuss two approaches of the backgrounds.

\section{Phenomenological background}

Since we are searching for spectral features which are distinct from the "smooth" background, it is reasonable to assume that the majority of the observational data can be fitted by the background $[49,66]$. Therefore a so-called "minimum model," as that employed in Ref. [12], is adopted. However, to better reproduce the wide-band data from $\mathrm{GeV}$ to multi-TeV, we slightly extend the "minimum model" with a low-energy break and a high-energy cutoff. In this case the background model is directly fitted to the data, without considering the propagation effect. ${ }^{1}$ The background model includes three components, the primary $e^{-}$, secondary $e^{+} e^{-}$, and a source term of $e^{+} e^{-}$[63], i.e.,

\footnotetext{
${ }^{1}$ For the DM component to be discussed later, the propagation is still included.
}

$$
\begin{gathered}
\phi_{e^{-}}=C_{e^{-}} E^{-\gamma_{1}^{e^{-}}}\left[1+\left(E / E_{\mathrm{br}}^{e^{-}}\right)^{\gamma_{2}^{e^{-}}}\right]^{-1} \exp \left(-E / E_{c}^{e^{-}}\right), \\
\phi_{e^{+}}=C_{e^{+}} E^{-\gamma_{1}^{e^{+}}}\left[1+\left(E / E_{\mathrm{br}}^{e^{+}}\right)^{\gamma_{2}^{e^{+}}}\right]^{-1} \\
\phi_{\mathrm{s}}=C_{\mathrm{s}} E^{-\gamma^{s}} \exp \left(-E / E_{\mathrm{c}}^{\mathrm{s}}\right) .
\end{gathered}
$$

The total background energy spectrum of $e^{-}+e^{+}$is then

$$
\phi_{\mathrm{bkg}, e^{ \pm}}=\phi_{e^{-}}+1.6 \phi_{e^{+}}+2 \phi_{\mathrm{s}}
$$

where the factor 1.6 is due to the asymmetry of the electron and positron productions in $p p$ collisions [67]. The positron fraction is

$$
f=\left(\phi_{e^{+}}+\phi_{\mathrm{s}}\right) / \phi_{\mathrm{bkg}, e^{ \pm}}
$$

The best-fit parameters are summarized in Table I. Note that they are somewhat different from that given in Ref. [63] because of different data sets used in the fitting. When the DM contribution is added in the model, we enable the backgrounds to vary to some degree through multiplying adjustment factors $\alpha_{i} E^{\beta_{i}}$, with $i=\left\{e^{-}, e^{+}, s\right\}$, on $\phi_{e}^{-}, \phi_{e}^{+}$, and $\phi_{\mathrm{s}}$, respectively [24]. Parameters $\alpha_{i}$ and $\beta_{i}$ are optimized during the fitting.

\section{Physical background}

A more physical way to calculate the background starts with the injection spectrum of different components of sources, and calculate the propagated spectra of them. The LIKEDM package is used to calculate the propagation of various components. For the injection spectrum of primary electrons, a three-segment broken power-law model with an exponential cutoff is assumed. The first break at several $\mathrm{GeV}$ is to account for the low-energy data, and the second break at several tens $\mathrm{GeV}$ is to explain the spectral hardening [68-70], and the cutoff is to reproduce the DAMPE high-energy data [57]. The injection spectrum of primary electrons is then

$$
\begin{aligned}
\Phi_{e^{-}}= & A_{e^{-}} E^{-\nu_{1}^{e^{-}}}\left[1+\left(E / E_{\mathrm{br} 1}^{e^{-}}\right)^{3}\right]^{\left(\nu_{1}^{e^{-}}-\nu_{2}^{e^{-}}\right) / 3} \\
& \times\left[1+\left(E / E_{\mathrm{br} 2}^{e^{-}}\right)^{3}\right]^{\left(\nu_{2}^{e^{-}}-\nu_{3}^{e^{-}}\right) / 3} \exp \left(-E / E_{c}^{e^{-}}\right) .
\end{aligned}
$$

TABLE I. Best-fit parameters of the backgrounds.

\begin{tabular}{lccccc}
\hline \hline & $\begin{array}{c}C \\
\left(\mathrm{GeV}^{-1} \mathrm{~m}^{-2} \mathrm{~s}^{-1} \mathrm{sr}^{-1}\right)\end{array}$ & $\gamma_{1}$ & $\gamma_{2}$ & $\begin{array}{c}E_{\mathrm{br}} \\
(\mathrm{GeV})\end{array}$ & $\begin{array}{c}E_{c} \\
(\mathrm{GeV})\end{array}$ \\
\hline$\phi_{e^{-}}$ & 21.3417 & 0.8380 & 2.4075 & 3.3391 & $1.4435 \times 10^{4}$ \\
$\phi_{e^{+}}$ & 1.1947 & 0.7138 & 2.5898 & 2.7479 & $\ldots$ \\
$\phi_{s}$ & 0.9799 & 2.3828 & $\ldots$ & $\ldots$ & 842.93 \\
\hline \hline
\end{tabular}


Here we use $\Phi$ to describe the injection spectrum, in order to distinguish from the propagated fluxes $\phi$ given in Eqs. (3)-(7).

The secondary positron spectrum from $p p$ collisions is calculated by the GALPROP code. This component only needs to be calculated once. A constant factor is multiplied to its flux during the fitting, which accounts for possible uncertainties of the theoretical prediction [71].

A pulsarlike component is also added in the model, which is expected to contribute to the positron and electron excesses. The injection spectrum of electrons and positrons from pulsars is described by an exponential cutoff powerlaw form

$$
\Phi_{\mathrm{psr}}=A_{\mathrm{psr}} E^{-\iota^{\mathrm{psr}}} \exp \left(-E / E_{c}^{\mathrm{psr}}\right) .
$$

The spatial distribution of pulsars is adopted to be the same as the primary cosmic ray source distribution [61]. Note that here we assume a continuously distributed pulsar population to account for the primary $e^{+} e^{-}$. It has been discussed extensively that nearby isolated pulsars may be important to contribute to the electron/positron fluxes around $\mathrm{TeV}$ energies [33,38-40]. Given that there are many free uncertain parameters in such a scenario, we simply assume a population of such sources in this work. The spectra of electrons and positrons from a series of sources are actually similar to that from a source population.

\section{RESULTS}

We use a maximum likelihood fitting method based on the MINUIT tool in ROOT to search for the DM component. The data used include the AMS-02 positron fraction [12], the AMS-02 total electron fluxes below $25 \mathrm{GeV}$ [16], and the DAMPE data [57]. The background parameters are optimized using the profile likelihood method. In this study, we adopt the Navarro-Frenk-White (NFW) DM density profile [72], which is

$$
\rho_{\mathrm{NFW}}(r)=\frac{\rho_{s}}{\left(\frac{r}{r_{s}}\right)\left(1+\frac{r}{r_{s}}\right)^{2}} .
$$

The profile parameters can be found in Table II of Ref. [73].

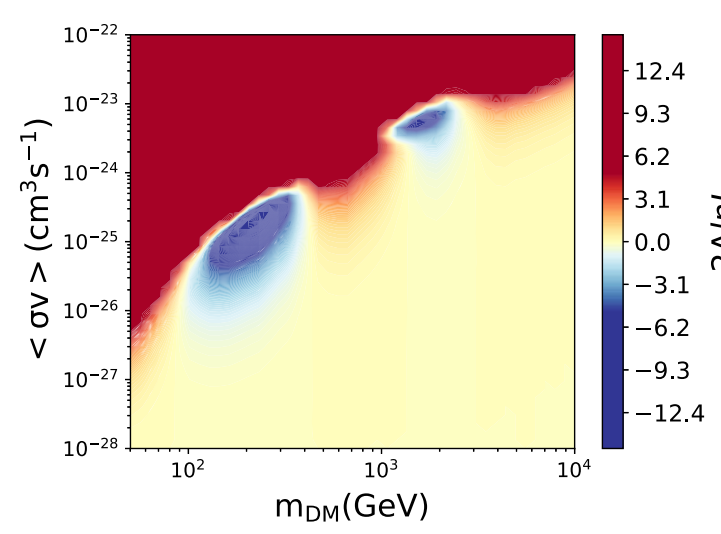

(a) $m_{\phi} / m_{\mathrm{DM}}=0.1$

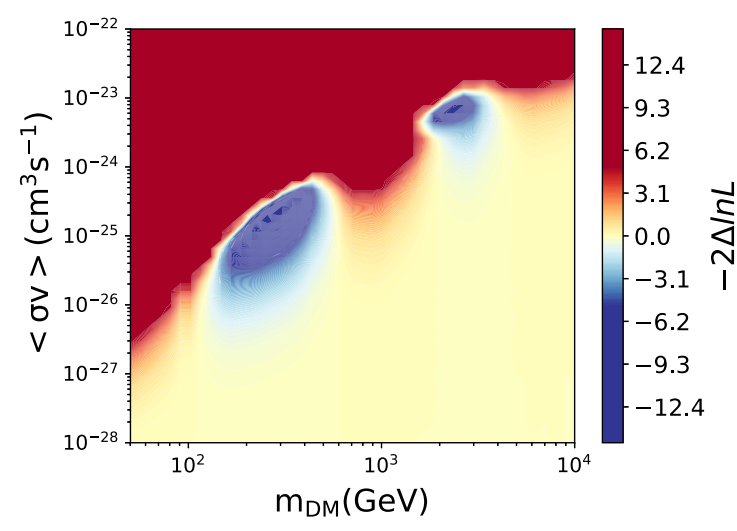

(c) $m_{\phi} / m_{\mathrm{DM}}=0.9$

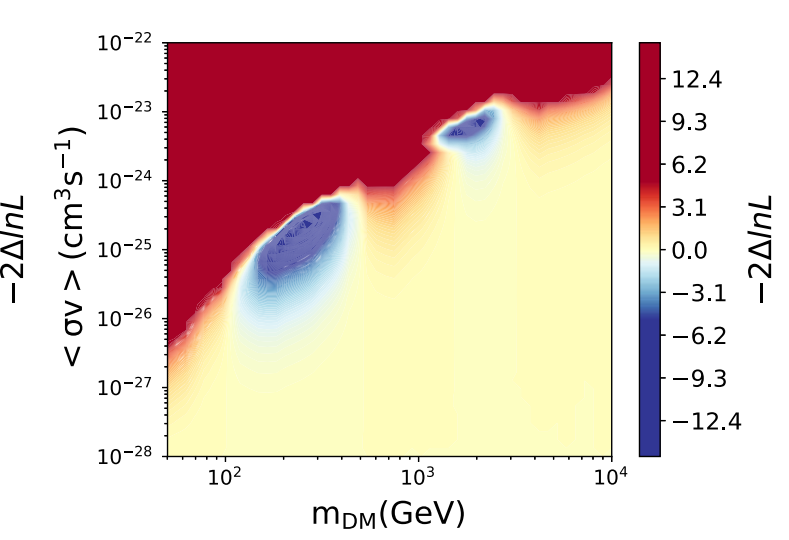

(b) $m_{\phi} / m_{\mathrm{DM}}=0.5$

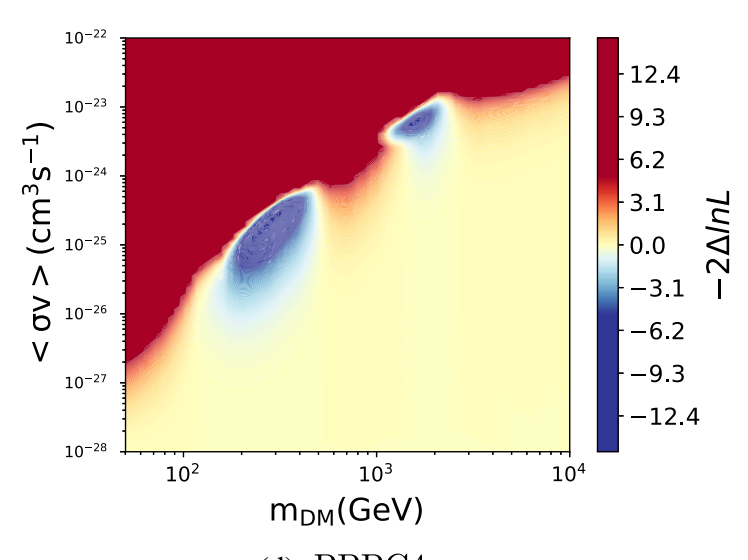

(d) PPPC4

FIG. 2. Log-likelihood maps on the $m_{\mathrm{DM}}-\langle\sigma v\rangle$ plane, for injection spectra with different mass ratios $\left(m_{\phi} / m_{\mathrm{DM}}=0.1,0.5,0.9\right)$ and the PPPC4 spectrum. The physical background is assumed. 

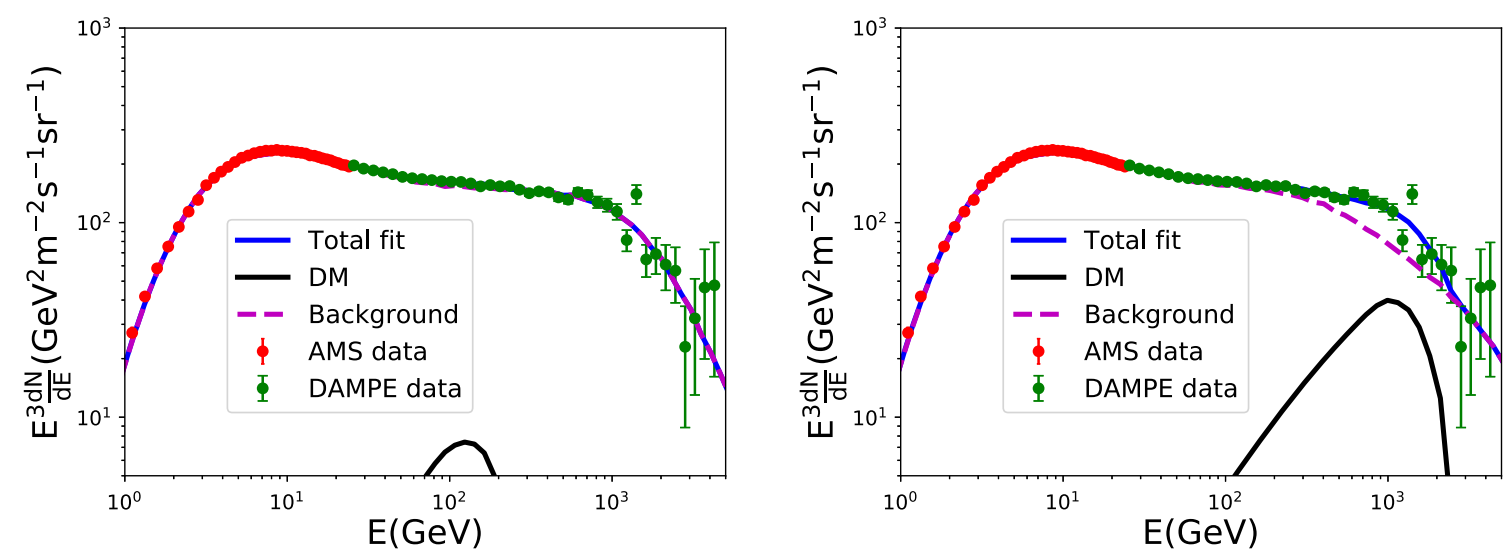

FIG. 3. Best-fit model prediction of the energy spectrum of electrons plus positrons, for $m_{\mathrm{DM}}=331 \mathrm{GeV}$, $\langle\sigma v\rangle=$ $2.0 \times 10^{-25} \mathrm{~cm}^{3} \mathrm{~s}^{-1}$ (left panel), and $m_{\mathrm{DM}}=2870 \mathrm{GeV}$ and $\langle\sigma v\rangle=7.9 \times 10^{-24} \mathrm{~cm}^{3} \mathrm{~s}^{-1}$ (right panel). In both cases, $m_{\phi} / m_{\mathrm{DM}}=$ 0.9 and the physical background are adopted. Also shown are the data of AMS-02 [16] and DAMPE [57].

\section{A. DM annihilation in the Milky Way halo}

We first consider the scenario that DM annihilates in the whole Milky Way halo. The maps of $-2 \Delta \ln \mathcal{L}=$ $-2\left(\ln \mathcal{L}-\ln \mathcal{L}_{0}\right)$, where $\mathcal{L}_{0}(\mathcal{L})$ is the likelihood without (with) the DM contribution, on the $\left(m_{\mathrm{DM}},\langle\sigma v\rangle\right)$ plane for $e^{+} e^{-}$injection spectra for different mass ratios $m_{\phi} / m_{\mathrm{DM}}$ and the PPPC4 energy spectrum are shown in Fig. 2. Here the physical background model is adopted. We find that there are two regions (blue in Fig. 2) with $m_{\mathrm{DM}} \sim$ (100-500) $\mathrm{GeV}$ and $\sim(2-3) \mathrm{TeV}$ are "favored" by the data. Both regions are due to the degeneracy between the DM contribution and the background (in particular, the pulsar contribution). To better understand this, we show in Fig. 3 the comparison of the total electron spectrum between the best-fit model and the data, for mass ratio of $m_{\phi} / m_{\mathrm{DM}}=0.9$ and $\left(m_{\mathrm{DM}},\langle\sigma v\rangle\right)=(331 \mathrm{GeV}$, $\left.2.0 \times 10^{-25} \mathrm{~cm}^{3} \mathrm{~s}^{-1}\right)$ (left panel) and $(2870 \mathrm{GeV}, 7.9 \times$ $10^{-24} \mathrm{~cm}^{3} \mathrm{~s}^{-1}$ ) (right panel). The low mass region is favored when the pulsar component has a very hard spectrum, which gives a "dip" on the spectrum and the addition of a DM component can somehow improve the fit. We have tested that if the pulsar injection spectrum is set to be softer than $E^{-1}$, this low mass region becomes much less significant. The same applies to the high mass region.

We also test the results for the phenomenological background model. We find that only the $\sim(2-3) \mathrm{TeV}$ region is slightly favored, with $-2 \Delta \ln \mathcal{L}$ about -2 . This is mainly because the phenomenological background model fits the data better than the physical model, and the left room for $\mathrm{DM}$ contribution is smaller. The (2-3) $\mathrm{TeV}$ region again reflects the degeneracy between the DM component and the $\phi_{s}$ component.

We thus give the $95 \%$ upper limits of the annihilation cross section for given DM mass $m_{\mathrm{DM}}$, through setting $-\ln \mathcal{L}(\langle\sigma v\rangle) \leq-\ln \mathcal{L}_{\max }+1.35$. The results are given in Fig. 4. The left panel is for the phenomenological background, and the right panel is for the physical background. Since the results depend on the background assumptions,
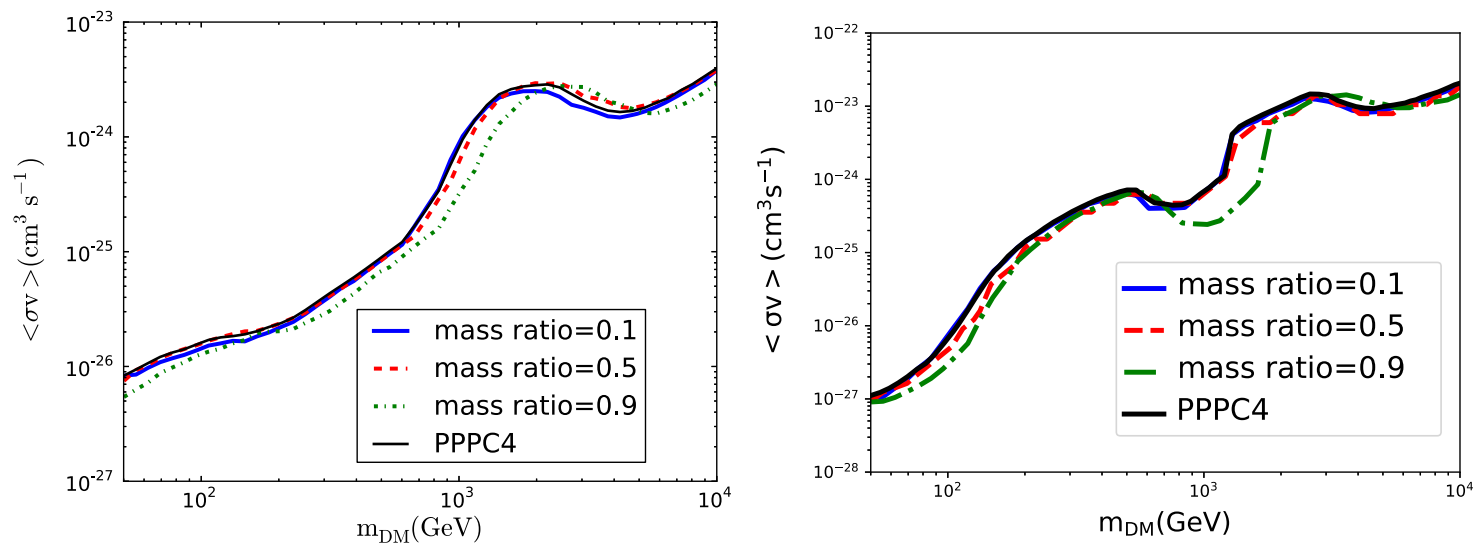

FIG. 4. The $95 \%$ confidence level upper limits on the DM annihilation cross section $\langle\sigma v\rangle$ as a function of $m_{\mathrm{DM}}$ for the phenomenological (left) and physical (right) background models. 


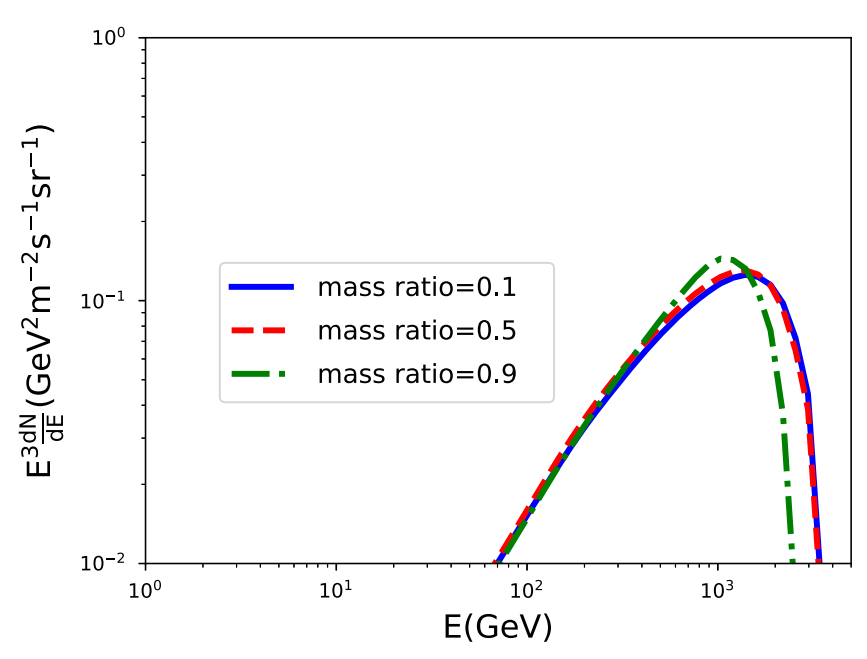

FIG. 5. Propagated spectra of electrons for $m_{\mathrm{DM}}=3000 \mathrm{GeV}$ and $m_{\phi} / m_{\mathrm{DM}}=0.1,0.5,0.9$.

we conservatively adopt the larger values as the final constraints. The upper limits range from $\sim 10^{-26} \mathrm{~cm}^{3} \mathrm{~s}^{-1}$ for $m_{\mathrm{DM}}=50 \mathrm{GeV}$ to $\sim 10^{-23} \mathrm{~cm}^{3} \mathrm{~s}^{-1}$ for $m_{\mathrm{DM}}=10 \mathrm{TeV}$. The results for different mass ratios do not differ significantly from each other. This is because the injection spectra have been broadened after the propagation, and their differences become smaller (see Fig. 5). For $m_{\phi} / m_{\mathrm{DM}}=$ 0.9 the constraints are slightly tighter than the others. This is expected since a higher mass ratio of $m_{\phi} / m_{\mathrm{DM}}$ gives a narrower injection $e^{+} e^{-}$spectrum (Fig. 1). The constraints become weaker for $m_{\mathrm{DM}} \sim 2 \mathrm{TeV}$ and $\sim 300 \mathrm{GeV}$, due to the "favored signal" regions in such mass ranges.

\section{B. DM annihilation in a local subhalo}

The DM annihilation into $e^{+} e^{-}$channel in a local DM subhalo was proposed to explain the tentative peak structure at $1.4 \mathrm{TeV}$ of the DAMPE data $[58,74,75]$. If the mass

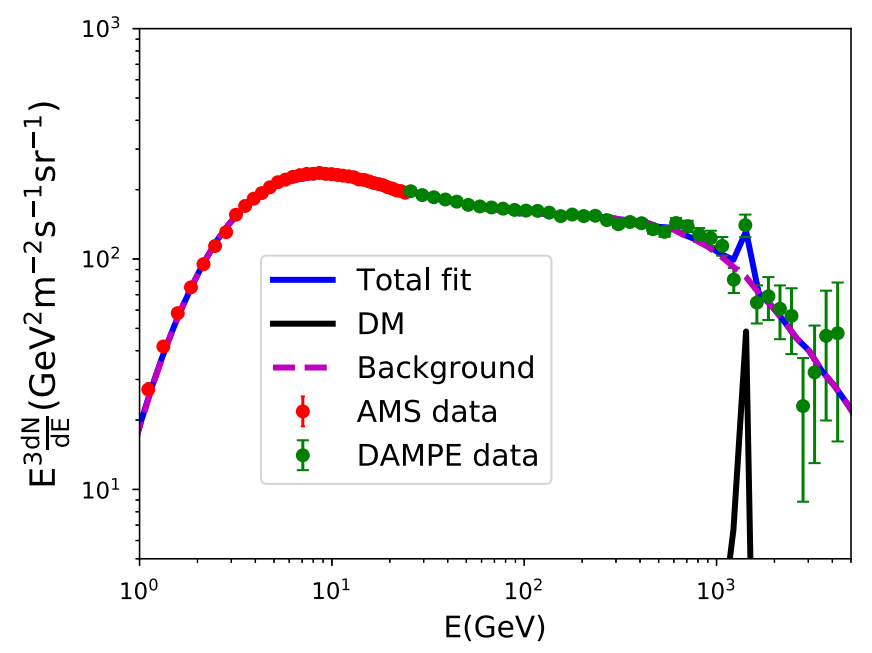

FIG. 7. Same as Fig. 3 but for a local DM subhalo scenario. Model parameters are $m_{\phi} / m_{\mathrm{DM}}=0.995, \quad m_{\mathrm{DM}}=3 \mathrm{TeV}$, $\langle\sigma v\rangle=3.4 \times 10^{-26} \mathrm{~cm}^{3} \mathrm{~s}^{-1}$, and the distance and mass of the subhalo are $0.1 \mathrm{kpc}$ and $1.9 \times 10^{7} \mathrm{M}_{\odot}$ [58].

ratio $m_{\phi} / m_{\mathrm{DM}}$ is very close to 1 , the resulting $e^{+} e^{-}$ spectrum from the DM annihilation will be nearly monochromatic. In this subsection, we examine whether such a narrow box-shaped $e^{+} e^{-}$spectrum can reasonably reproduce the data.

Figure 6 shows the log-likelihood maps for such a scenario, assuming a subhalo mass of $1.9 \times 10^{7} \mathrm{M}_{\odot}$ and a distance of $0.1 \mathrm{kpc}$ [58]. The left panel is for $m_{\phi} / m_{\mathrm{DM}}=0.5$, and the right panel is for $m_{\phi} / m_{\mathrm{DM}}=$ 0.995 . We find that for a relatively small mass ratio between the intermediate particle and the DM, the addition of the DM component always makes the fit worse. If the mass ratio is close to 1 , the fit to the data can be improved. The best-fit result is presented in Fig. 7 and the corresponding DM model parameters are $m_{\phi} / m_{\mathrm{DM}}=0.995$, $m_{\mathrm{DM}}=3 \mathrm{TeV}$, and $\langle\sigma v\rangle=3.4 \times 10^{-26} \mathrm{~cm}^{3} \mathrm{~s}^{-1}$.

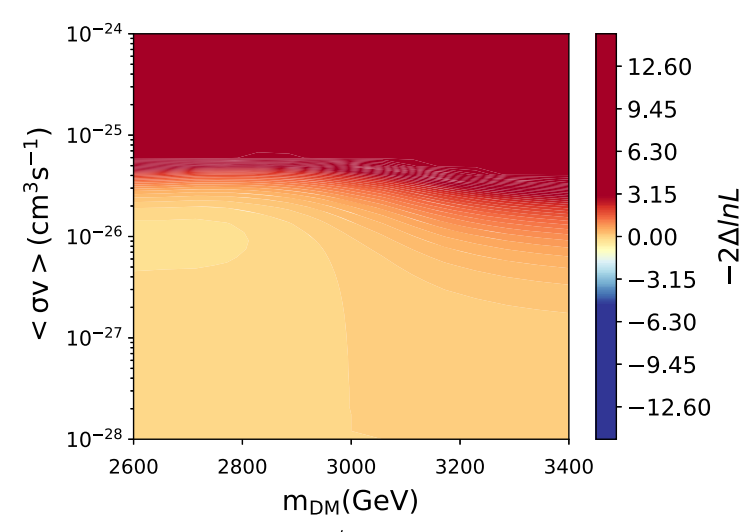

(a) $m_{\phi} / m_{\mathrm{DM}}=0.5$

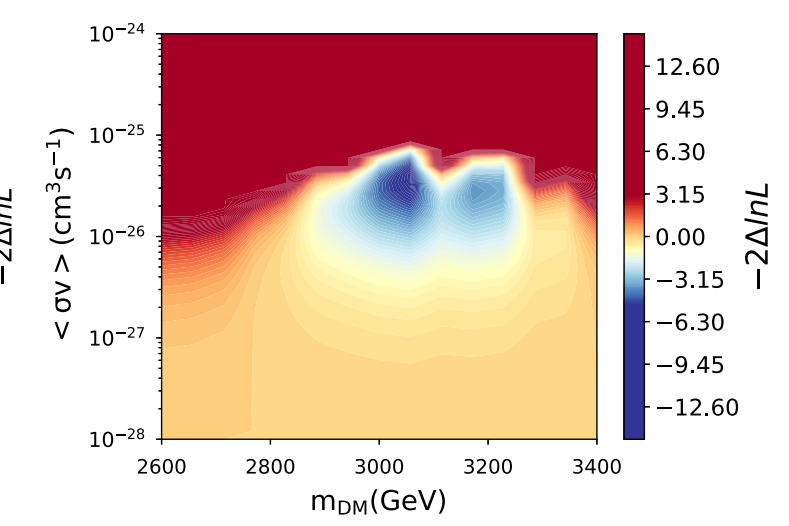

(b) $m_{\phi} / m_{\mathrm{DM}}=0.995$

FIG. 6. Log-likelihood maps on the $\left(m_{\mathrm{DM}},\langle\sigma v\rangle\right)$ plane in the DM subhalo scenario, for a mass ratio of $m_{\phi} / m_{\mathrm{DM}}=0.5$ (left) and 0.995 (right). The distance of the subhalo is $0.1 \mathrm{kpc}$, and its mass is $1.9 \times 10^{7} \mathrm{M}_{\odot}$. 


\section{CONCLUSION}

In this work, we have investigated a specific model in which DM particles initially annihilate into a pair of intermediate particles which then decay into electrons and positron. The resulting electron/positron spectrum has a boxlike shape (before propagation), which may be observed in cosmic ray electron and positron data. Using the AMS-02 and DAMPE data, we have searched for such a possible signal.

We have considered two types of background models, one is a phenomenological model based on the assumption that the majority of the shallow spectrum comes from astrophysical sources, and the other is a physically motivated model. For the physical background model, we find that the data tend to "favor" the DM particles with $m_{\mathrm{DM}} \sim(100-500) \mathrm{GeV}$ and $\sim(2-3) \mathrm{TeV}$. However, we found that such results are simply due to the degeneracy between the DM component and the pulsar component, and hence do not really point toward the existence of DM particles. Therefore the $95 \%$ upper limits of the DM annihilation cross section have been derived. The constraints range from $\sim 10^{-26} \mathrm{~cm}^{3} \mathrm{~s}^{-1}$ to $\sim 10^{-23} \mathrm{~cm}^{3} \mathrm{~s}^{-1}$ for DM mass from $50 \mathrm{GeV}$ to $10 \mathrm{TeV}$.
Within the scenario of a local DM subhalo, we find that a high mass ratio between $m_{\phi}$ and $m_{\mathrm{DM}}$ (for which the resulting $e^{+} e^{-}$spectrum is linelike) can improve the fit to the tentative peak at $1.4 \mathrm{TeV}$. DAMPE was designed to work for 3 years [56]. Given its current perfect status, it is expected to work for at least 5 years. By then, the statistics of electrons and positrons recorded by DAMPE will increase by several times. A more accurate spectrum may shed further light on the indirect detection of DM annihilation.

\section{ACKNOWLEDGMENTS}

This work is supported in part by the National Key Research and Development Program of China (No. 2016YFA0400200), National Natural Science of China (No. 11525313, No. 11722328, No. 11773075, and No. U1738206), the Youth Innovation Promotion Association of Chinese Academy of Sciences (No. 2016288), the Natural Science Foundation of Jiangsu Province (No. BK20151608), and the 100 Talents program of Chinese Academy of Sciences.
[1] P. J. Fox, R. Harnik, J. Kopp, and Y. Tsai, Phys. Rev. D 85, 056011 (2012).

[2] J. Goodman, M. Ibe, A. Rajaraman, W. Shepherd, T. M. P. Tait, and H.-B. Yu, Phys. Rev. D 82, 116010 (2010).

[3] D. S. Akerib et al., Phys. Rev. Lett. 112, 091303 (2014).

[4] J. Angle et al., Phys. Rev. Lett. 100, 021303 (2008).

[5] J. L. Feng, K. T. Matchev, and F. Wilczek, Phys. Rev. D 63, 045024 (2001).

[6] M. Ackermann et al., Phys. Rev. Lett. 107, 241302 (2011).

[7] G. Bertone, D. Hooper, and J. Silk, Phys. Rep. 405, 279 (2005).

[8] L. Bergstrom, Rep. Prog. Phys. 63, 793 (2000).

[9] G. Bertone, Particle Dark Matter: Observations, Models and Searches (Cambridge University Press, Cambridge, England, 2010).

[10] L. Bergstrom, arXiv:1202.1170 (2012).

[11] O. Adriani et al., Nature (London) 458, 607 (2009).

[12] M. Aguilar et al., Phys. Rev. Lett. 110, 141102 (2013).

[13] L. Accardo et al., Phys. Rev. Lett. 113, 121101 (2014).

[14] J. Chang et al., Nature (London) 456, 362 (2008).

[15] M. Ackermann et al., Phys. Rev. Lett. 108, 011103 (2012).

[16] M. Aguilar et al., Phys. Rev. Lett. 113, 221102 (2014).

[17] O. Adriani et al., Phys. Rev. Lett. 102, 051101 (2009).

[18] O. Adriani et al., Phys. Rev. Lett. 105, 121101 (2010).

[19] M. Aguilar et al., Phys. Rev. Lett. 117, 091103 (2016).

[20] M.-Y. Cui, Q. Yuan, Y.-L. S. Tsai, and Y.-Z. Fan, Phys. Rev. Lett. 118, 191101 (2017).

[21] A. Cuoco, M. Kramer, and M. Korsmeier, Phys. Rev. Lett. 118, 191102 (2017).
[22] L. Bergström, T. Bringmann, and J. Edsjö, Phys. Rev. D 78, 103520 (2008).

[23] V. Barger, W.-Y. Keung, D. Marfatia, and G. Shaughnessy, Phys. Lett. B 672, 141 (2009).

[24] M. Cirelli, M. Kadastik, M. Raidal, and A. Strumia, Nucl. Phys. B813, 1 (2009); 873, 530(A) (2013).

[25] P.-F. Yin, Q. Yuan, J. Liu, J. Zhang, X.-J. Bi, S.-H. Zhu, and X. Zhang, Phys. Rev. D 79, 023512 (2009).

[26] J. Zhang, X.-J. Bi, J. Liu, S.-M. Liu, P.-F. Yin, Q. Yuan, and S.-H. Zhu, Phys. Rev. D 80, 023007 (2009).

[27] Q. Yuan, X.-J. Bi, G.-M. Chen, Y.-Q. Guo, S.-J. Lin, and X. Zhang, Astropart. Phys. 60, 1 (2015).

[28] J. Kopp, Phys. Rev. D 88, 076013 (2013).

[29] I. Cholis and D. Hooper, Phys. Rev. D 88, 023013 (2013).

[30] H.-B. Jin, Y.-L. Wu, and Y.-F. Zhou, J. Cosmol. Astropart. Phys. 11 (2013) 026.

[31] H. Yüksel, M. D. Kistler, and T. Stanev, Phys. Rev. Lett. 103, 051101 (2009).

[32] D. Hooper, P. Blasi, and P. D. Serpico, J. Cosmol. Astropart. Phys. 01 (2009) 025.

[33] S. Profumo, Central Eur. J. Phys. 10, 1 (2012).

[34] D. Malyshev, I. Cholis, and J. Gelfand, Phys. Rev. D 80, 063005 (2009).

[35] H.-B. Hu, Q. Yuan, B. Wang, C. Fan, J.-L. Zhang, and X.-J. Bi, Astrophys. J. Lett. 700, L170 (2009).

[36] P. Blasi, Phys. Rev. Lett. 103, 051104 (2009).

[37] T. Linden and S. Profumo, Astrophys. J. 772, 18 (2013).

[38] P.-F. Yin, Z.-H. Yu, Q. Yuan, and X.-J. Bi, Phys. Rev. D 88, 023001 (2013). 
[39] M. Di Mauro, F. Donato, N. Fornengo, R. Lineros, and A. Vittino, J. Cosmol. Astropart. Phys. 04 (2014) 006.

[40] K. Fang, B.-B. Wang, X.-J. Bi, S.-J. Lin, and P.-F. Yin, Astrophys. J. 836, 172 (2017).

[41] N. Arkani-Hamed, D. P. Finkbeiner, T. R. Slatyer, and N. Weiner, Phys. Rev. D 79, 015014 (2009).

[42] A. Lopez, C. Savage, D. Spolyar, and D. Q. Adams, J. Cosmol. Astropart. Phys. 03 (2016) 033.

[43] A. Scaffidi, K. Freese, J. Li, C. Savage, M. White, and A. G. Williams, Phys. Rev. D 93, 115024 (2016).

[44] M. Papucci and A. Strumia, J. Cosmol. Astropart. Phys. 03 (2010) 014.

[45] G. Bertone, M. Cirelli, A. Strumia, and M. Taoso, J. Cosmol. Astropart. Phys. 03 (2009) 009.

[46] L. Bergstrom, G. Bertone, T. Bringmann, J. Edsjo, and M. Taoso, Phys. Rev. D 79, 081303 (2009).

[47] T. R. Slatyer, N. Padmanabhan, and D. P. Finkbeiner, Phys. Rev. D 80, 043526 (2009).

[48] T. R. Slatyer, Phys. Rev. D 93, 023527 (2016).

[49] L. Bergstrom, T. Bringmann, I. Cholis, D. Hooper, and C. Weniger, Phys. Rev. Lett. 111, 171101 (2013).

[50] J. Mardon, Y. Nomura, D. Stolarski, and J. Thaler, J. Cosmol. Astropart. Phys. 05 (2009) 016.

[51] G. Elor, N. L. Rodd, and T. R. Slatyer, Phys. Rev. D 91, 103531 (2015).

[52] X.-J. Huang, C.-C. Wei, Y.-L. Wu, W.-H. Zhang, and Y.-F. Zhou, Phys. Rev. D 95, 063021 (2017).

[53] A. Ibarra, S. L. Gehler, and M. Pato, J. Cosmol. Astropart. Phys. 07 (2012) 043.

[54] S. Li, Y.-F. Liang, Z.-Q. Xia, L. Zu, K.-K. Duan, Z.-Q. Shen, L. Feng, Q. Yuan, and Y.-Z. Fan, Phys. Rev. D 97, 083007 (2018).

[55] J. Chang, Chin. J. Space Sci. 34, 550 (2014).

[56] J. Chang et al., Astropart. Phys. 95, 6 (2017).
[57] G. Ambrosia et al., Nature 552, 63 (2017).

[58] Q. Yuan et al., arXiv:1711.10989.

[59] M. Cirelli, G. Corcella, A. Hektor, G. Hutsi, M. Kadastik, P. Panci, M. Raidal, F. Sala, and A. Strumia, J. Cosmol. Astropart. Phys. 03 (2011) 051.

[60] T. Sjostrand, S. Mrenna, and P. Z. Skands, Comput. Phys. Commun. 178, 852 (2008).

[61] A. W. Strong and I. V. Moskalenko, Astrophys. J. 509, 212 (1998).

[62] C. Evoli, D. Gaggero, D. Grasso, and L. Maccione, J. Cosmol. Astropart. Phys. 10 (2008) 018.

[63] X. Huang, Y.-L. S. Tsai, and Q. Yuan, Comput. Phys. Commun. 213, 252 (2017).

[64] M. Ackermann et al. (LAT Collaboration), Astrophys. J. 750, 3 (2012).

[65] C. S. Shen, Astrophys. J. Lett. 162, L181 (1970).

[66] A. Ibarra, A. S. Lamperstorfer, and J. Silk, Phys. Rev. D 89, 063539 (2014).

[67] T. Kamae, N. Karlsson, T. Mizuno, T. Abe, and T. Koi, Astrophys. J. 647, 692 (2006).

[68] L. Feng, R.-Z. Yang, H.-N. He, T.-K. Dong, Y.-Z. Fan, and J. Chang, Phys. Lett. B 728, 250 (2014).

[69] Q. Yuan and X.-J. Bi, Phys. Lett. B 727, 1 (2013).

[70] X. Li, Z.-Q. Shen, B.-Q. Lu, T.-K. Dong, Y.-Z. Fan, L. Feng, S.-M. Liu, and J. Chang, Phys. Lett. B 749, 267 (2015).

[71] S.-J. Lin, Q. Yuan, and X.-J. Bi, Phys. Rev. D 91, 063508 (2015).

[72] J. F. Navarro, C. S. Frenk, and S. D. M. White, Astrophys. J. 490, 493 (1997).

[73] G. Bertone, M. Cirelli, A. Strumia, and M. Taoso, J. Cosmol. Astropart. Phys. 03 (2009) 009.

[74] X.-J. Huang, Y.-L. Wu, W.-H. Zhang, and Y.-F. Zhou, Phys. Rev. D 97, 091701 (2018).

[75] H.-B. Jin et al., arXiv:1712.00362. 